\title{
Hypocholesterolemia, Hypertriglyceridemia, Suicide, and Suicide Ideation in Children Hospitalized for Psychiatric Diseases
}

\author{
CHARLES J. GLUECK, FRANK E. KULLER, TRACEY HAMER, ROGER RODRIGUEZ, \\ FELIX SOSA, LUANN SIEVE-SMITH, AND JOHN A. MORRISON
}

Cholesterol Center, Jenish Hospital; Children's Hospital; and Millcreek Psychiatric Center for Children, Cincinnati, Ohio 45229

\begin{abstract}
To assess relationships of total plasma cholesterol (TC) and triglyceride (TG) values to suicide, suicide ideation, and hospitalization for psychiatric disease, we studied 220 children, ages 5 to $18 \mathrm{y}$, hospitalized with affective, adjustment, disruptive, anxiety, schizophrenic, other, and organic psychiatric disorders. The 135 male and 85 female patients had higher TG values $(p=0.0001$ and 0.0003 , respectively) and higher Quetelet Indices ( $p=$ 0.0001 and 0.003 , respectively) than the 732 male and 316 female schoolchild controls; male patients had higher TC values than male controls $(p=0.014)$. Substance abuse in patients was an independent inverse determinant of 'TC value ( $p=0.05)$; TG value correlated positively with alcohol use $(p \leq 0.1)$ and sustance abuse $(p<0.05)$. After covariance adjustment for age, race, sex, and Quetelet, children having adjustment disorders with depression had much lower covariance-adjusted TC value than control schoolchildren (3.91 versus $4.29 \mathrm{mmol} / \mathrm{l}, p=0.003$ ), whereas those with disruptive behavior with oppositional defiant disorder had much higher adjusted TC value $\mathbf{( 5 . 0 9}$ $\mathrm{mmol} / \mathrm{L}, p=0.0001$ ). After covariance adjusting for age, race, sex, Quetelet, cigarette smoking, alcohol use, and substance abuse, children having adjustment disorders with concomitant depression had the highest group suicide tendencies (attempts and ideation) and the lowest covarianceadjusted TC value $(4.03 \mathrm{mmol} / \mathrm{L})$. Conversely, children having disruptive behavior with attention deficit hyperactivity disorder or disruptive behavior with oppositional defiant disorder had $50 \%$ lower suicide index than those with adjustment disorders with concomitant depression and higher adjusted TC levels $(4.45$ and $5.12 \mathrm{mmol} / \mathrm{L}, p=$ 0.0003 ). Children with psychiatric hospitalizations have high TG values, and those with adjustment disorders with concomitant depression have low TC values, which is positively associated with suicide tendencies. We speculate that the associations between low TC level, high TG level, suicide tendency, and hospitalization for psychiatric disease do not reflect cause and effect but may be epiphenomena. (Pediatr Res 35: 602-610, 1994)
\end{abstract}

\section{INTRODUCTION}

In prospective epidemiologic studies, individuals with plasma total cholesterol values in the lowest quartile (1) or less than 4.14

Received June 16, 1993; accepted December 10, 1993.

Correspondence and reprint requests: Charles J. Glueck, M.D., Cholesterol Center, 3200 Burnet Avenue, Cincinnati, OH 45229.

Supported in part by Grants 732 and 2-728 from the Jewish Hospital Medical Research Council, Jewish Hospital of Cincinnati.
mmol/L (2, 3) have experienced greater nonillness mortality than those with average or high cholesterol levels. There is also evidence from metaanalysis of randomized, controlled, primary prevention trials of cholesterol lowering that treated men had more suicide and trauma deaths (nonillness mortality) than controls (4-6). However, Wysowski and Gross (7) have challenged the contention that cholesterol lowering might be causally related to nonillness mortality in primary prevention trials $(5$, 6 ). They reported that the study subjects with nonillness mortality often had a history of alcohol abuse or emotional problems or had been so noncompliant with regard to the study medication as to achieve little to no cholesterol lowering (7). In aggregate, the epidemiologic (1-3) and controlled clinical trial (4-7) associations of low or lowered serum cholesterol with increased nonillness mortality are currently poorly understood, but they have substantial public health implications (4).

Low serum cholesterol values have been reported in association with various psychopathologic conditions, such as schizophrenia (8) and affective disorders (9-11), and is seen in criminals with diagnoses of violent or aggressive conduct disorders (1214). None of the studies of the associations of low cholesterol level with psychopathologic conditions $(8-14)$, however, have accounted for diet, alcohol, or substance abuse. Psychopathologic disorders (8-14) may be associated with malnutrition or alcoholic liver failure, conditions that often lower plasma cholesterol, or with substance abuse, for which there is as yet no body of evidence as to cholesterol- or triglyceride-altering effect.

Recently, we studied hypocholesterolemia in 203 patients hospitalized because of affective disorders compared with 1595 selfreferred subjects in an urban supermarket screening $(9,10)$. Low plasma cholesterol value $(<4.14 \mathrm{mmol} / \mathrm{L})$ was four to 10 times more common in patients with affective disorders than in supermarket screenees $(9,10)$. We postulated that undernutrition, alcohol dependence, or both as a consequence of affective disorders may induce hypocholesterolemia, thus in part accounting for the reported inverse association of plasma cholesterol value with suicide deaths in prospective epidemiologic studies $(1-3)$. Morgan et al. (11) carried out a prospective cohort study of 1020 adult white men to assess the relationship of mood to plasma cholesterol levels. In men ages 70 and older, symptoms of depression were more common in the group with plasma cholesterol values below $4.14 \mathrm{mmol} / \mathrm{L}$, and depressive symptoms correlated inversely with plasma cholesterol. These findings persisted after adjustments for age, health status, weight loss, and change in cholesterol value during the preceding 13 y (11).

High triglyceride values may also be a determinant of symptoms of depression $(15,16)$. Recently, we studied 23 patients referred to the Jewish Hospital Cholesterol Center in Cincinnati for diagnosis and therapy of severe hypertriglyceridemia [typically $>11.3 \mathrm{mmol} / \mathrm{L}(15,16)]$. At study entry, $61 \%$ of the patients with hypertriglyceridemia had a normal Beck Depression Index 
score $(<10)(17), 22 \%$ had a score of $10-18$ (mild to moderate depressive symptoms), $13 \%$ had a moderate to severe score (between 19 and 29), and $4 \%$ had severe depressive symptoms $(\geq 30)$ (16). After major (46\%) lowering of plasma triglyceride values in these patients with diet plus Lopid $(15,16)$, highly significant reductions occurred in the Beck Depression Index scores. The greater the reduction in triglyceride value, the greater the reduction in the Beck Depression Index. Fowkes et al. (18) evaluated 1600 adult men and women with the Bedford Foulds personality deviance scale. They observed a positive correlation of serum triglyceride values with hostile acts and domineering attitude in both sexes (18). Whether the neurobehavioral correlates of high plasma triglyceride levels $(15,16,18)$ overlap or are distinct from those associated with low plasma cholesterol levels is presently unknown (4).

Suicides, homicides, and accidents taken together are the leading cause of death for persons between the ages of 1 to $39 y$ in the United States (19-21). Suicide is currently the third leading cause of death for young people between the ages of 15 and 24 y (22) and has become the second leading cause of morbidity in this age group (23). Major risk factors for adolescent suicide include depression, alcohol and substance abuse, family conflict, and conduct disturbance $(23,24)$. Estimates for actual suicide attempts in children and young adults are $3 \%$ for elementary students, $11 \%$ for high school students, and 15 to $18 \%$ for college students (25).

Because suicide is a major public health problem in children and young adults (19-25), because low plasma cholesterol values have been associated with increased nonillness mortality (1-6), and because low cholesterol or high triglyceride values have been associated with psychopathologic disorders (12-14) and with symptoms of depression $(9-11,15,16,18)$, our specific aim in the current study was to assess relationships of plasma cholesterol and triglyceride values to suicide, suicide ideation, and hospital- ization for psychiatric disease in 220 children from the ages of 5 to $18 \mathrm{y}$.

\section{MATERIALS AND METHODS}

Pationts. With no selection bias, we examined 220 patients' charts, comprising all pediatric admissions to the Millcreek Psychiatric Center for Children in Cincinnati, Ohio, from January 1, 1992, to March 31, 1993. This work observed a protocol approved by the State of Ohio Psychiatric Hospital Board.

Control children. Healthy control children $(n=1048)$ came from three longitudinal studies of coronary heart disease risk factors in schoolchildren. They included 1 ) boys from 13 greater Cincinnati area parochial schools, 2) boys and girls from nine public elementary schools in the biracial, suburban Princeton School District, and 3) girls from public and parochial schools in Hamilton County. These studies were carried out from 1984 to 1992. The children were recruited by grade in school without any selection bias (race, sex, socioeconomic status, family history of cardiovascular disease, for example). The Boys' Maturation Study, a longitudinal evaluation of sexual maturation and lipids, contributed 517 control boys, ages $10-16$ y, from the 13 greater Cincinnati parochial schools. Their participation rate was $74 \%$. The second group contributing to the control schoolchildren included 215 boys and 234 girls, ages 8-13 (grades 3-7) at entry to the Princeton Fitness 2000 project, a program of nutritional modification. Their participation rate was $60 \%$. The third group contributing to our controls, 82 girls, ages 10-16 y, came from Hamilton County public and parochial schoolgirls who were members of school classes from which children were recruited for the National Growth and Health Study but who were more than 1 wk too young $(<8.98 \mathrm{y})$ or 1 wk too old $(>11.02 \mathrm{y})$ for that study. Their participation rate was $75 \%$. Thus, our schoolchild controls encompassed a variegated biracial group with a

Table 1. Least square means (by analysis of covariance), unadjusted means, and medians of total plasma cholesterol and triglyceride (mmol/L) values by psichiatric diagnosis groups and subgroups in 220 pediatric psychiatric inpatients*

\begin{tabular}{|c|c|c|c|c|c|c|c|c|}
\hline \multirow[b]{3}{*}{ Subgroup } & \multirow[b]{3}{*}{ Diagnosis group } & \multirow[b]{3}{*}{$n$} & \multicolumn{3}{|c|}{$\mathrm{TC}$} & \multicolumn{3}{|c|}{ TG } \\
\hline & & & \multirow{2}{*}{$\begin{array}{l}\text { Adjusted } \\
\text { LS Mean }\end{array}$} & \multicolumn{2}{|c|}{ Unadjusted } & \multirow{2}{*}{$\begin{array}{l}\text { Adjusted } \\
\text { LS Mean }\end{array}$} & \multicolumn{2}{|c|}{ Unadjusted } \\
\hline & & & & Mean & Median & & Mean & Median \\
\hline \multirow[t]{5}{*}{$I$} & Affective disorders & 71 & $4.55 \dagger$ & 4.50 & 4.37 & 1.32 & 1.31 & 1.13 \\
\hline & I-1 Bipolar & 20 & $4.58 \ddagger$ & 4.58 & 4.58 & 1.54 & 1.48 & 1.15 \\
\hline & 1-2 Major depression & 19 & 4.50 & 4.40 & 4.40 & 1.20 & 1.20 & 1.16 \\
\hline & I-3 Dysthymia & 18 & $4.63 \ddagger$ & 4.55 & 4.32 & 1.17 & 1.17 & 0.97 \\
\hline & $\begin{array}{l}\text { I-4 Depressive NOS } \\
\text { (I-4') Schizoaffectiveş }\end{array}$ & $\begin{array}{r}11 \\
3\end{array}$ & 4.58 & 4.45 & 4.37 & 1.30 & 1.31 & 1.10 \\
\hline \multirow[t]{3}{*}{ II } & Adjustment disorders & 64 & $4.16+$ & 4.19 & 4.14 & 1.24 & 1.25 & 1.03 \\
\hline & II-1 With depression & 37 & $4.03 \ddagger$ & 3.98 & 3.83 & 1.25 & 1.25 & 0.98 \\
\hline & 11-2 Others & 27 & $4.37 \|$ & 4.47 & 4.55 & 1.23 & 1.24 & 1.10 \\
\hline \multirow[t]{4}{*}{ III } & Disruptive behavior & 58 & 4.47 & 4.55 & 4.50 & 1.29 & 1.29 & 1.12 \\
\hline & III-1 ADHD & 24 & $4.45 \pi$ & 4.53 & 4.22 & 1.33 & 1.28 & 0.95 \\
\hline & III-2 Conduct disorder & 19 & $4.34^{* *}$ & 4.27 & 4.50 & 1.26 & 1.32 & 1.32 \\
\hline & III-3 Oppositional defiant & 15 & $5.12 \ddagger\|\|^{* *}$ & 5.20 & 5.20 & 1.22 & 1.30 & 1.38 \\
\hline IV & Anxiety disorders & 12 & 4.76 & 4.63 & 4.71 & 1.22 & 1.14 & 0.89 \\
\hline V & Other & 6 & 4.47 & 4.29 & 4.16 & 0.90 & 1.24 & 1.04 \\
\hline VI & Schizophrenia & 5 & 5.02 & 4.99 & 4.99 & 1.12 & 1.13 & 1.10 \\
\hline VII & Organic & 4 & 4.58 & 4.60 & 4.73 & 1.42 & 1.38 & 0.60 \\
\hline
\end{tabular}

* Covariance adjusted for age, race, sex, Quetelet Index, cigarette smoking, alcohol use, and substance abuse. $p$ values taken from analysis of covariance. TC, total plasma cholesterol value; TG, triglyceride value; LS, least square; NOS, not otherwise specified; ADHD, attention deficit hyperactivity disorder.

† Group II differs from group I $(p=0.016)$.

‡ Group II-1 differs from groups I-1, I-3, and III-3 ( $p=0.03,0.03$, and 0.0003 , respectively).

$\S$ Three schizoaffective children included in the 71 with affective disorders, but because $n$ was only three, they were not included in the nine subgroups.

\| Group II-2 differs from group III-3 $(p=0.02)$.

I Group III-1 differs from group III-3 $(p=0.03)$.

** Group III-2 differs from group III-3 $(p=0.02)$. 


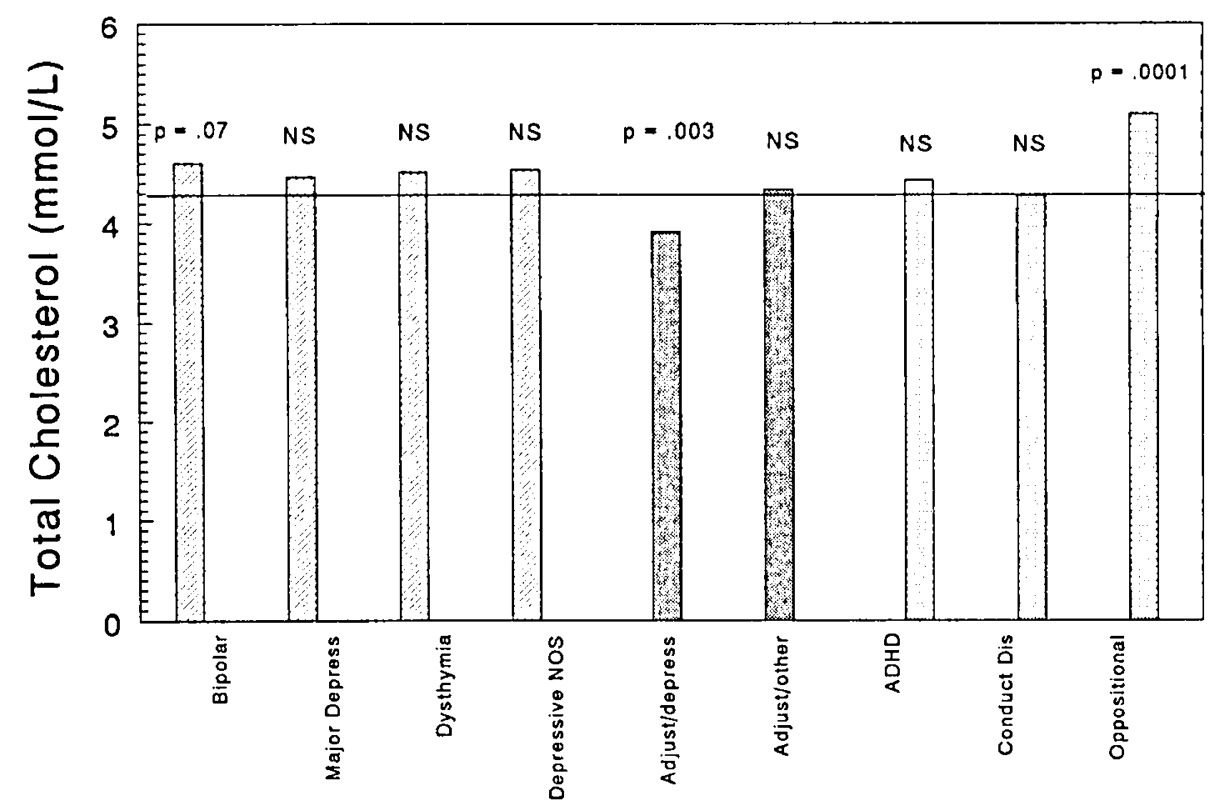

Fig. 1. Comparison of plasma total cholesterol levels after covariance adjustment for age, race, sex, and Quetelet Index; nine psychiatric diagnostic subgroups are displayed [bipolar, major depression (major depress), dysthmia, depression not otherwise specified (depressive NOS), adjustment disorder with depression (adjust/depress), adjustment disorder (other) (adjust/other) attention deficit hyperactivity disorder (ADHD), conduct disorder (conduct dis), and oppositional defiant (oppositional)] vs healthy schoolchild controls.

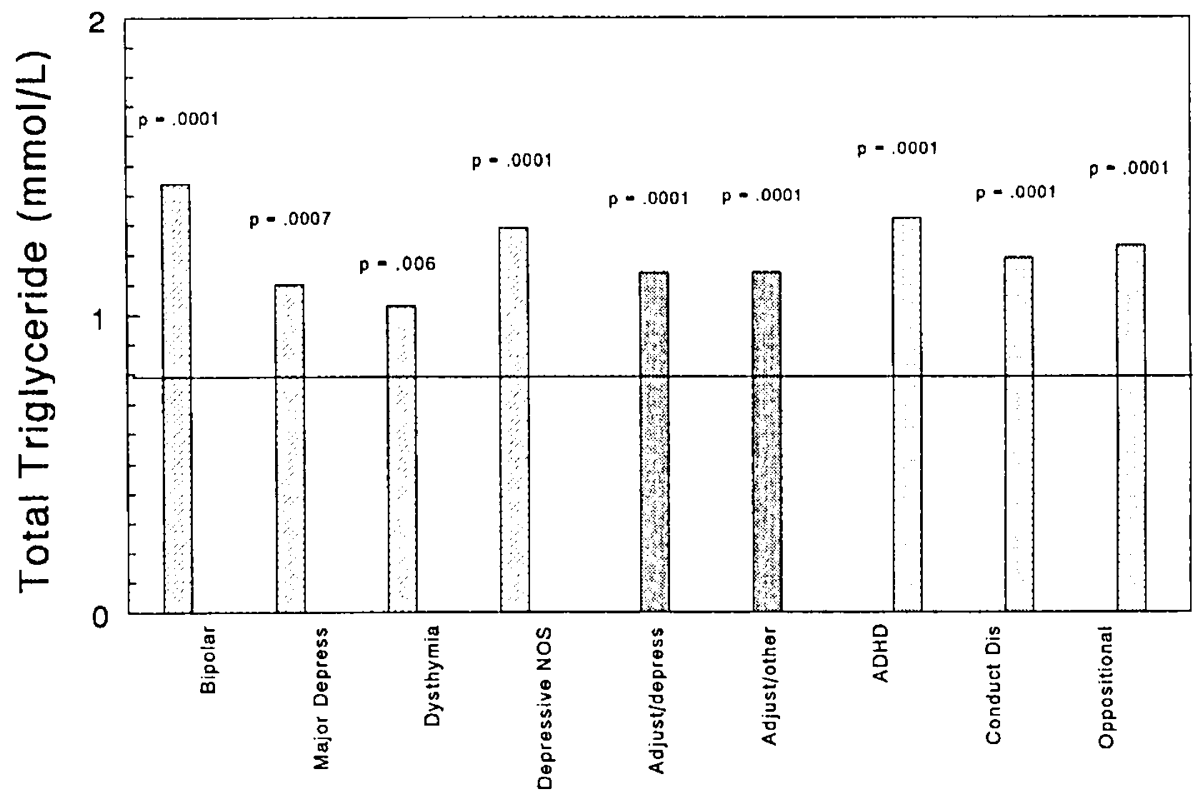

= Control Group

Fig. 2. Comparison of triglyceride levels after covariance adjustment for age, race, sex, and Quetelet Index; nine psychiatric diagnostic subgroups vs healthy schoolchild controls. See legend to Figure 1 for explanation of subgroups.

wide range of socioeconomic status from the greater Cincinnati metropolitan area. Participation rates were primarily dependent on the level of grant support for staff for student recruitment, sampling, and rerecruitment efforts for nonparticipants. In the Princeton Fitness 2000 study $(n=449)$, participants and nonparticipants did not differ $(p>0.1)$ with regard to age and Quetelet Index. Comparisons of fasting plasma cholesterol and triglyceride levels between participant and nonparticipant control schoolchildren could not be conducted because nonparticipants did not have lipid profiles.

Information on age, race, sex, height, and weight was obtained in the three subgroups of control schoolchildren. No questions about substance abuse or prescription medications were asked. Interviewers collected information on smoking and alcohol intake only in the 517 control boys in the parochial schools and not in the other control schoolchildren. Because we did not have smoking or alcohol data for the remaining 531 schoolchild controls, we chose not to present partial smoking or alcohol data for controls.

Lipids and lipoprotein cholesterol levels were measured in a Lipid Research Clinics standardized laboratory for the 1048 schoolchildren after a 12-h fast. The psychiatric inpatients' cho- 
Table 2. Univariate determinants of cholesterol and triglyceride values in 220 children (Spearman correlation coefficients)

\begin{tabular}{lrl}
\hline \multicolumn{1}{c}{ Whole group } & $r$ value & $p$ value \\
\hline Total cholesterol & & \\
$\quad$ Correlation pair & & \\
$\quad$ Substance abuse & -0.17 & $\leq 0.01$ \\
$\quad$ Race* & 0.16 & $\leq 0.02$ \\
Triglyceride & & \\
Quetelet Index & 0.40 & $\leq 0.001$ \\
Alcohol & 0.17 & $\leq 0.01$ \\
Alcohol amount & 0.19 & $\leq 0.01$ \\
Substance abuse & 0.14 & $\leq 0.05$ \\
Cigarette smoking & 0.24 & $\leq 0.001$ \\
Cigarette amount & 0.25 & $\leq 0.001$ \\
\hline
\end{tabular}

* White $=1$, black $=2$.

Table 3. Multivariate determinants of plasma cholesterol and triglyceride values (Stepwise regression)*

\begin{tabular}{clccccc}
\hline \multirow{2}{*}{$\begin{array}{c}\text { Dependent } \\
\text { variable }\end{array}$} & \multicolumn{1}{c}{$\begin{array}{c}\text { Explanatory } \\
\text { variable }\end{array}$} & $\begin{array}{c}\text { Standardized } \\
\text { regression } \\
\text { coefficient }\end{array}$ & & \multicolumn{2}{c}{ Model } \\
\cline { 4 - 7 } & TC & Substance abuse & -0.14 & 0.05 & 0.05 & 0.006 \\
& Race & 0.14 & 0.047 & & \\
TG & Cigarettes & 22.7 & 0.027 & 0.10 & 0.0004 \\
& Race & -20.6 & 0.033 & & \\
& Quetelet index & 15.4 & 0.026 & & \\
& Sex & -18.1 & 0.05 & & \\
\hline
\end{tabular}

*Whole group, $n=202$. Model: $\mathrm{TC}=$ age, $\operatorname{sex}($ males $=1$, females $=$ 2), alcohol, cigarettes, substance abuse, Quetelet, and race (whites $=1$, blacks $=2)$. TG $=$ Age, sex $($ males $=1$, females $=2)$, alcohol, cigarettes. substance abuse, Quetelet, and race ( whites $=1$, blacks $=2$ ).

lesterol and triglyceride determinations were performed (after a 12-h fast) in a separate College of American Pathologists but non-Lipid Research Clinics standardized laboratory. However, the same enzymatic methods (9) and standards were used for measurement of plasma total cholesterol and triglyceride values in the psychiatric inpatient and schoolchild control cohorts, and they provided comparable results. HDL and LDL cholesterol values were not measured in psychiatric inpatients because of limited funds for patient studies.

Clinical data acquisition in patients. Axis I and axis II admitting diagnoses were recorded for each patient. We also recorded the data that had been systemically obtained by hospital staff interviews and by laboratory services on admission for all children, as follows: l) fasting plasma cholesterol and triglyceride values; 2) height, weight, and calculated Quetelet Index [ $(\mathrm{kg} /$ $\left.\mathrm{cm}^{2}\right) \times 1000$ ]; 3 ) cigarette smoking (yes, no, and if yes, number of cigarettes per day); 4) alcohol intake (yes, no, and if yes, number of beverages per day); 5) history of suicide attempt or suicide ideation; 6) medication use, broadly grouped in the following categories: antidepressants, antihypertensives, mood stabilizers, neuroleptics, and stimulants; and 7) current history of substance abuse (marijuana, crack, cocaine, heroin, amphetamines, barbiturates, and LSD, for example). Information regarding substance abuse, alcohol, and smoking was obtained by interviewers at the time of psychiatric hospital admission. Questions about substance abuse focused on current substance abuse (primarily in the week before admission).

Statistical analyses. To compare healthy control schoolchildren $(n=1048)$ to the 220 psychiatric inpatient children, we used analysis of covariance $(26,27)$. Plasma cholesterol and triglyceride values were adjusted for age, Quetelet Index, and race, with subsequent comparison of least square means [generated by analysis of covariance $(26,27)]$. Also, Quetelet Indices were adjusted for age and race, with subsequent comparison of least square means $(26,27)$. Both sets of data were gathered separately for boys and girls.
To further compare patients versus control children, we matched 361 controls to patients by sex, race, and age $( \pm 1 \mathrm{y})$. After determining the 361 controls' 10th percentile for cholesterol level and the 90th percentiles for triglyceride value and Quetelet Index, these cutpoints were then used to determine whether the observed percentages of patients falling below the controls' 10th percentile for cholesterol value or above the controls' 90th percentile for triglyceride value or Quetelet Index were more than those expected ( $\chi^{2}$ analysis).

Analysis of covariance $(26,27)$ was used to compare fasting plasma cholesterol and triglyceride values among psychiatric diagnosis subgroups, with only $p$ values $\leq 0.03$ displayed because multiple comparisons were made (Table 1). Arbitrarily, statistical analyses were performed only in subgroups with 10 patients or more (Table 1). The data for psychiatric diagnosis subgroups with less than 10 patients are presented for descriptive purposes only (Table 1). Plasma cholesterol and triglyceride levels in the psychiatric diagnosis subgroups were covariance adjusted for age, sex, race, Quetelet Index, alcohol use, smoking, and substance abuse, with subsequent comparison of least square means (Table $1)$, which were generated by analysis of covariance $(26,27)$.

Analysis of covariance $(26,27)$ was used to compare plasma cholesterol and triglyceride levels in control children with patients in the nine major psychiatric diagnosis subgroups after covariance adjusting for age, sex, race, and Quetelet Index (Figs. 1 and 2). Because much of the data were not normally distributed, nonparametric univariate Spearman correlations were calculated $(26,27)$ (Table 2).

Multivariate determinants of plasma cholesterol and triglyceride values were assessed by stepwise regression analysis $(26,27)$ with explanatory variables including age, sex, race, Quetelet Index, alcohol use, cigarette smoking, and substance abuse (Table 3 ). A significant model $p$ value indicates that some linear functions of the parameters in the model are significantly different from zero (Table 3$)(26,27)$.

To evaluate whether differences existed between psychiatric diagnosis groups for suicide tendency (suicide attempts and ideation) versus no suicide tendency, we performed logistic regression after covariance adjusting for age, sex, race, Quetelet Index, alcohol use, smoking, and substance abuse, with group differences assessed by maximum likelihood analysis $(26,27)$.

\section{RESULTS}

Cholesterol and triglyceride values and Quetelet Index. The 220 inpatient children with psychiatric disease included 135 boys (46 black, 85 white, four other) and 85 girls ( 24 black, 56 white, five other). The cohort of 1048 control schoolchildren included 732 boys ( 432 black, 300 white), and 316 girls ( 203 black, 113 white). Of the 220 patients, $64 \%$ were white, which was not different from the $61 \%$ of the 1048 controls who were white $(p$ $>0.01)$. Boys comprised $61 \%$ of patients and $70 \%$ of controls $(p=0.013)$.

Mean (SD) ages for male patients and controls were $12.4 \pm$ 3.3 and $12.9 \pm 2.7 \mathrm{y}$, respectively $(p<0.03)$, and for female patients and controls they were $13.1 \pm 3.1$ and $10.5 \pm 1.9 \mathrm{y}$, respectively $(p \leq 0.0001)$. Least square mean (SEM) plasma cholesterol values (covariance adjusted for age, race, and Quetelet Index) were higher $(4.43 \pm 0.067 \mathrm{mmol} / \mathrm{L})$ in male patients $(n$ $=135)$ than in male controls $(n=732)(4.25 \pm 0.028 \mathrm{mmol} / \mathrm{L}$, $p=0.014)$, as were triglyceride values $(1.28 \pm 0.042$ versus 0.77 $\pm 0.017 \mathrm{mmol} / \mathrm{L}, p=0.0001)$. Least square mean Quetelet Indices (covariance adjusted for age and race) were higher in male patients than in male controls $(2.17 \pm 0.04$ versus $2.007 \pm$ $0.16 \mathrm{mmol} / \mathrm{L}, p=0.0001)$. Least square means derived from analysis of covariance were nearly identical to the raw, unadjusted means for cholesterol and triglyceride values (Table 1).

Least square mean (SEM) plasma cholesterol values (covariance adjusted for age, race, and Quctelet Index) did not differ in female patients $(n=85)$ versus female controls $(n=316)(4.38$ 


\section{RACE}

WHITE $\square$ BLACK OTHER UNK

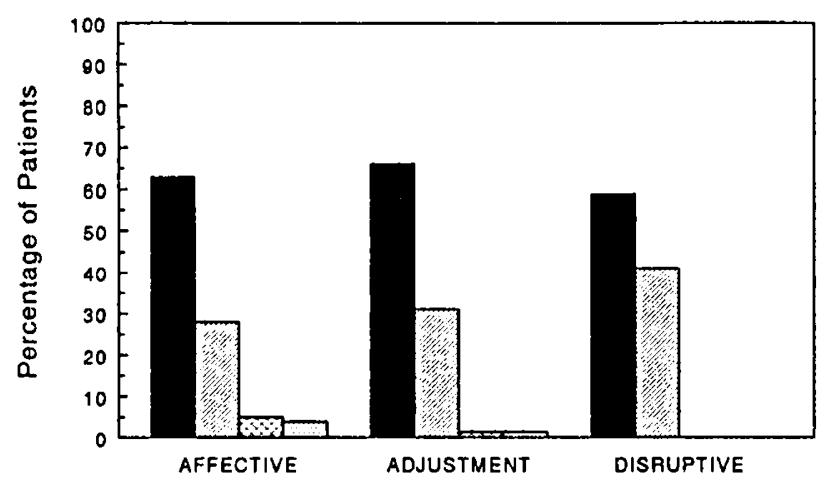

AGE

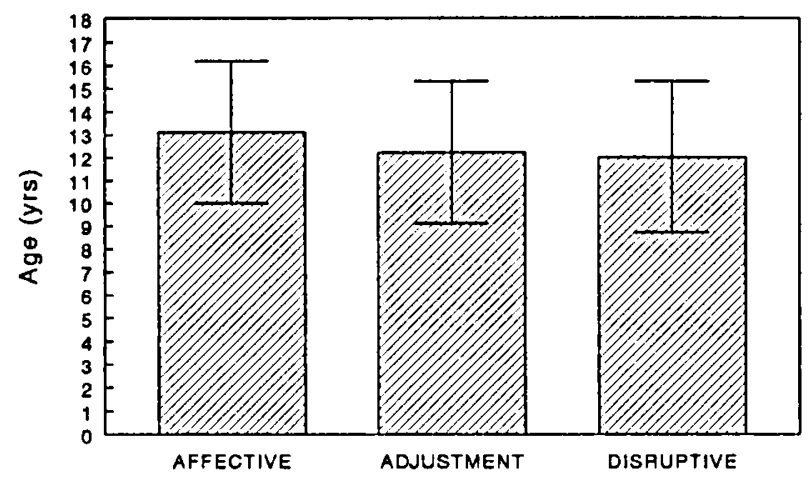

GENDER

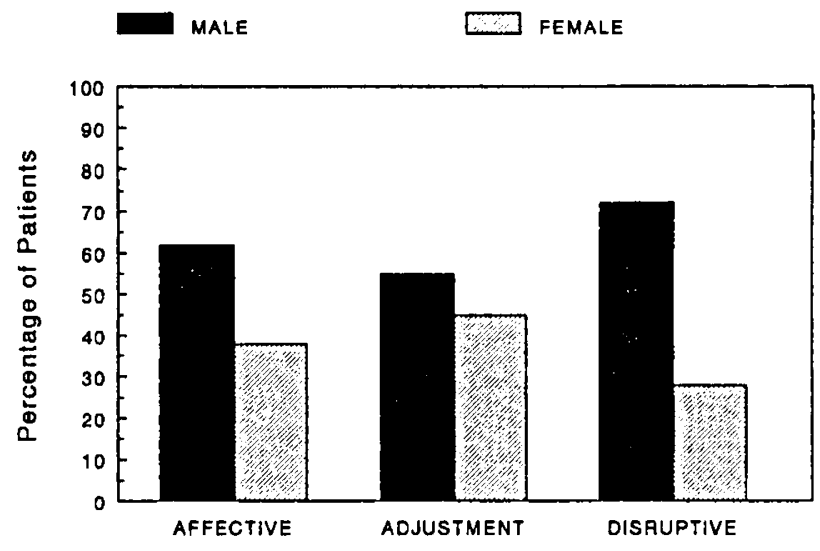

QUETELET

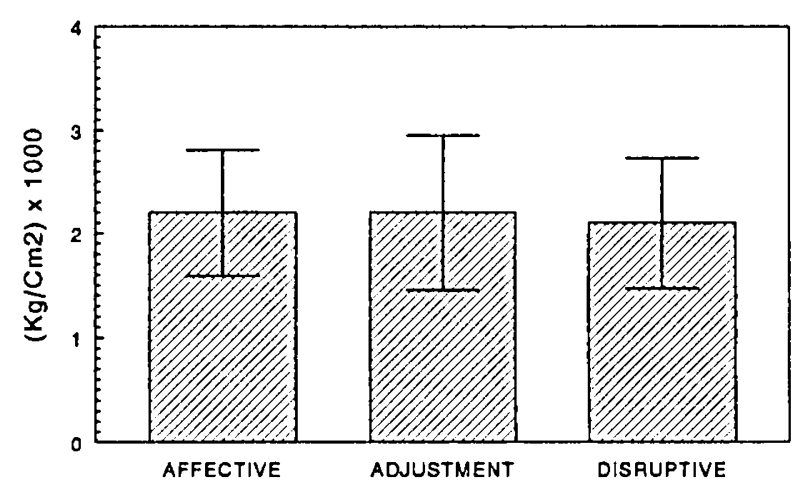

Fig. 3. Patients in the three major psychiatric diagnosis groups (affective disorders, adjustment disorders, and disruptive behavior) by race, gender, mean \pm SD age $(y)$, and mean \pm SD Quetelet Index.

\pm 0.093 versus $4.39 \pm 0.043 \mathrm{mmol} / \mathrm{L}, p=0.92$ ). However, female patients had higher covariance-adjusted triglyceride values $(1.08 \pm 0.05$ versus $0.88 \pm 0.022 \mathrm{mmol} / \mathrm{L}, p=0.0003)$ and higher Quetelet Indices $(2.12 \pm 0.05$ versus $1.95 \pm 0.02, p=$ 0.003).

The unadjusted plasma cholesterol 10th percentile values in male and female controls matched to male and female patients by race and age were 3.28 and $3.44 \mathrm{mmol} / \mathrm{L}$, respectively, the 90 th percentile triglyceride values were 1.30 and $1.41 \mathrm{mmol} / \mathrm{L}$, and the 90th percentile Quetelet Indices were 2.47 and 2.77. With these cutpoints for patients, the bottom decile plasma cholesterol value (present in $7.5 \%$ of boys and $17 \%$ of girls) was not overrepresented $(p>0.1)$, but top decile triglyceride values were markedly overrepresented in patients $[35 \%$ of boys $(p<$ $0.0001), 27 \%$ of girls $(p=0.002)]$. Top decile Quetelet Indices were overrepresented in male patients $(21 \%, p=0.03)$ but not female patients $(17 \%, p=0.15)$.

Psychiatric diagnoses in inpatient children. There were seven classes of axis I admitting psychiatric diagnoses, including three large groups: affective disorders $(n=71)$, adjustment disorders $(n=64)$, and disruptive behavior disorders $(n=58)$ (Table 1). Four smaller axis I diagnosis groups included anxiety disorders $(n=12)$, other disorders $(n=6)$, schizophrenia $(n=5)$, and organic disorders $(n=4)$ (Table 1$)$.

Of the 71 patients with affective disorders, there were four subgroups of patients: bipolar depression, major depression, dysthymia, and depressive disorders not otherwise specified (Table 1). Of the 64 children with adjustment disorders, there were two subgroups: adjustment disorders with depression and all others (Table 1). Subgrouped under the broad diagnosis of disruptive behavior were attention deficit hyperactivity disorders, conduct disorders, and oppositional defiant disorders (Table 1).

Characteristics of psychiatric inpatients in three largest psychiatric diagnosis groups. Children in the three largest psychiatric primary diagnosis groups (affective disorders, adjustment disorders, and disruptive behavior) did not differ by race, gender, alcohol abuse, substance abuse, age, or Quetelet Index $(p \geq 0.1$ for each) (Figs. 3 and 4). By $\chi^{2}$ analyses, children in the affective disorders group differed from the adjustment and disruptive groups by virtue of being on more prescription medications (Fig. $\left.4 ; \chi^{2}=7.22, p=0.027\right)$.

Plasma cholesterol and triglyceride levels related to psychiatric diagnoses. After covariance adjusting for age, race, sex, Quetelet Index, cigarette smoking, alcohol use, and substance abuse, adjusted plasma triglyceride levels did not differ $(p>0.10)$ among the seven major psychiatric diagnostic groups or among the nine diagnostic subgroups of the three largest psychiatric groups (Table 1).

Among the three largest broad psychiatric diagnosis categories, the lowest unadjusted mean and median cholesterol values and the lowest covariance-adjusted mean cholesterol levels were observed in the 64 children with adjustment disorders (Table 1). Children with adjustment disorders had lower adjusted mean cholesterol value $(4.16 \mathrm{mmol} / \mathrm{L})$ than those with affective disorders (4.55 mmol/L, $(p=0.016$; Table 1).

Of the nine major subgroups of the three largest psychiatric 
ALCOHOL ABUSERS

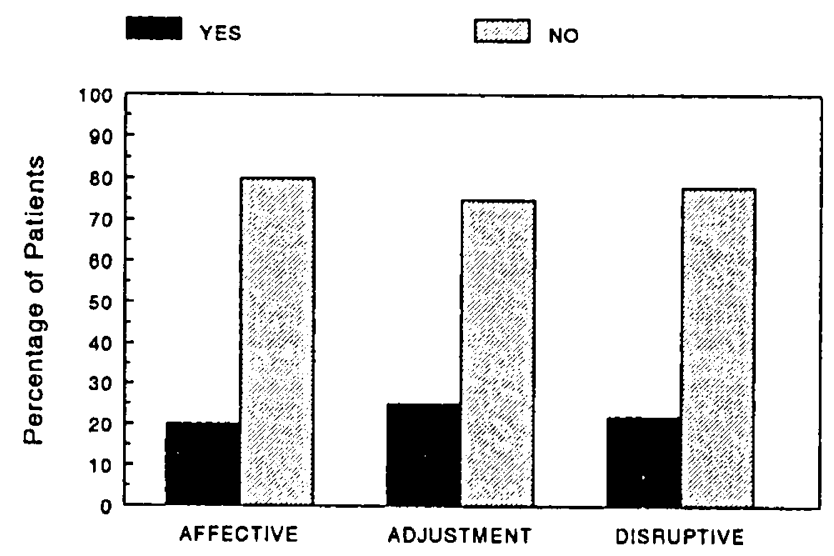

SUBSTANCE ABUSERS

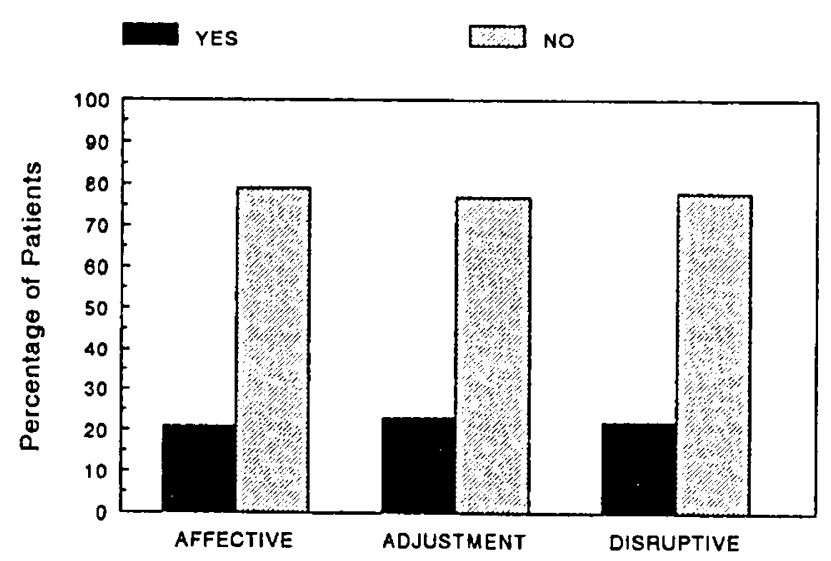

\section{ON PRESCRIPTION MEDICATION}

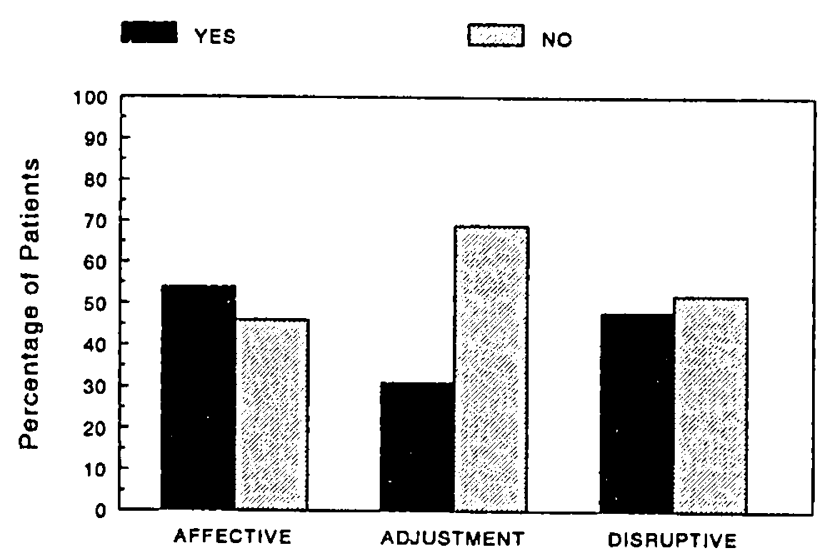

Fig. 4. Patients in the three major psychiatric diagnosis groups (affective disorders, adjustment disorders, and disruptive behavior) by percentage of patients as alcohol abusers, substance abusers, or those taking prescription medication.

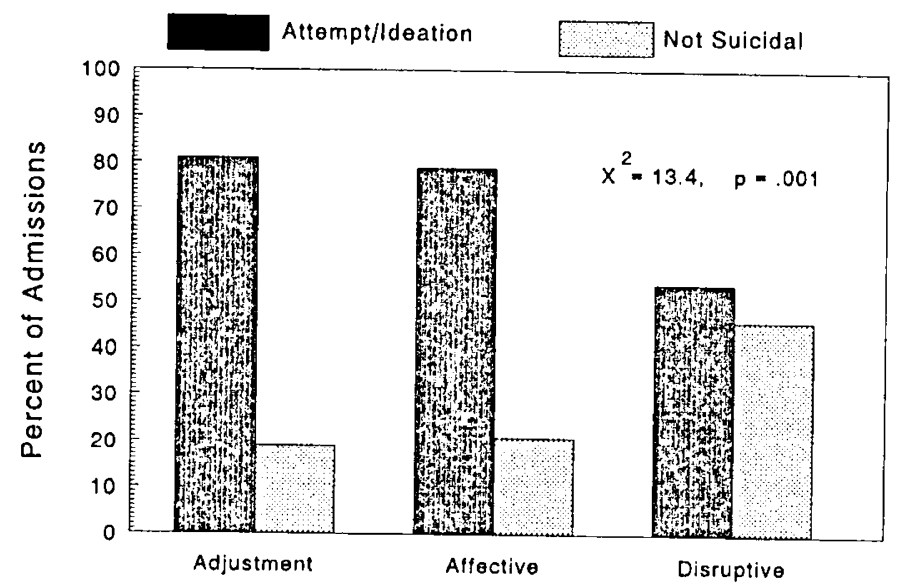

Fig. 5. Percentage of admissions with suicide attempts and ideation and nonsuicidal admissions in children with adjustment disorders, affective disorders, and disruptive disorders.

diagnosis groups, the lowest least square mean total cholesterol value $(4.03 \mathrm{mmol} / \mathrm{L})$ was seen in the 37 children who had adjustment disorders with depression (Table 1). Conversely, the highest least square mean total cholesterol value $(5.12 \mathrm{mmol} / \mathrm{L})$ was seen in the 15 children with oppositional defiant disorders (disruptive behavior) (Table 1). The 37 children having adjustment disorders with depression had lower $(p=0.03)$ adjusted total cholesterol values $(4.03 \mathrm{mmol} / \mathrm{L})$ than children with bipolar affective disorders $(4.58 \mathrm{mmol} / \mathrm{L})$, dysthymia $(4.63 \mathrm{mmol} / \mathrm{L}, p$ $=0.03$ ), and oppositional defiant disorders ( $5.12 \mathrm{mmol} / \mathrm{L}, p=$ 0.0003 ) (Table 1). Children with oppositional defiant disorders also had higher covariance-adjusted cholesterol values $\mathbf{5 . 1 2}$ $\mathrm{mmol} / \mathrm{L})$ than children with conduct disorders $(4.34 \mathrm{mmol} / \mathrm{L}, p$ $=0.02)$, all other adjustment disorders $(4.37 \mathrm{mmol} / \mathrm{L}, p=0.02)$, and attention deficit hyperactivity disorders $(4.45 \mathrm{mmol} / \mathrm{L}, p=$ 0.03) (Table 1).

After covariance adjusting for age, race, sex, and Quetelet Index, least square mean adjusted plasma total cholesterol (Fig. 1) and triglyceride (Fig. 2) values in the nine major psychiatric subgroups were compared with controls. The cholesterol levels in the 37 children having adjustment disorders with depression were much lower than those in schoolchild controls $(p=0.003$, Fig. 1), whereas the cholesterol levels were much higher in the 15 children with oppositional defiant disruptive behavior than in controls $(p=0.0001$, Fig. 1). Triglyceride levels in the nine major psychiatric subgroups were much higher than those in controls after covariance adjusting for age, sex, race, and Quetelet Index (Fig. 2).

Univariate and multivariate determinants of plasma total cholesterol and triglyceride values. The total cholesterol value was inversely associated with substance abuse $(p \leq 0.01)$ and posi- 

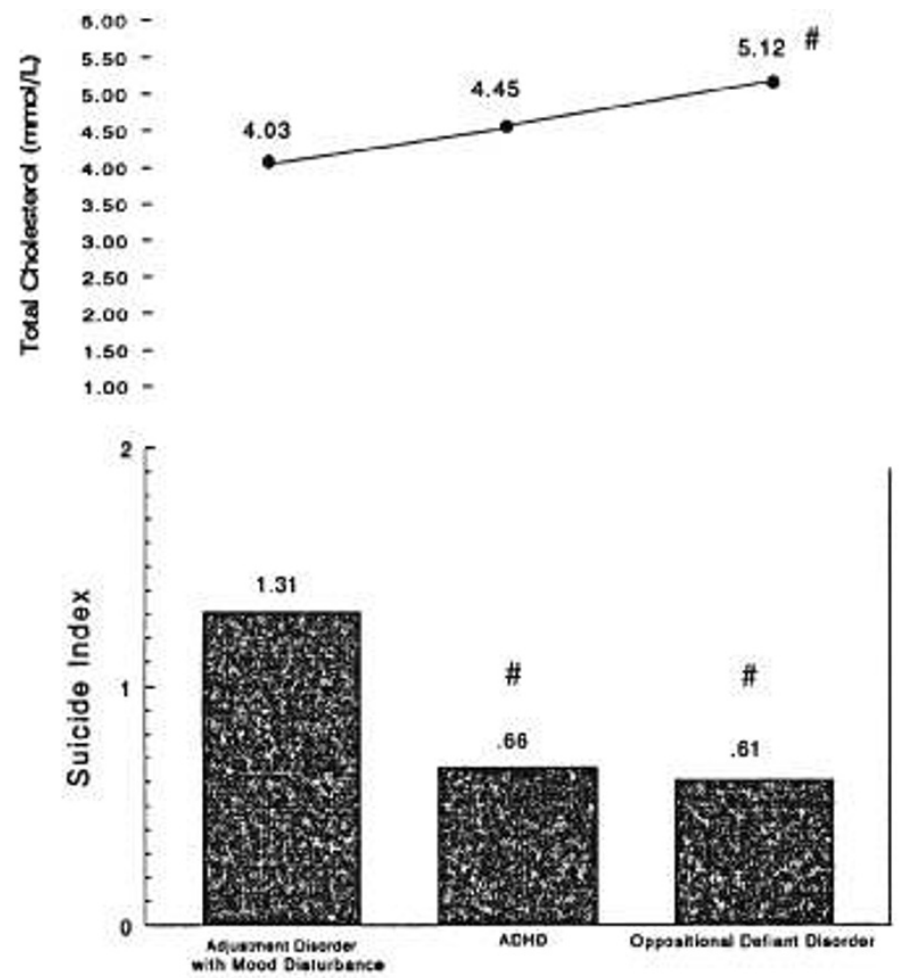

Fig. 6. Total cholesterol levels and suicide tendencies by subgroup psychiatric diagnosis, with concurrent subgroup least square mean total plasma cholesterol levels $(\mathrm{mmol} / \mathrm{L})$ after covariance adjustment for age; race; sex; Quetelet; and cigarette, alcohol, and substance abuse. Suicide index: $(0=$ not suicidal, $1=$ suicide ideation, $2=$ suicide attempt $)$. $A D H D$, attention deficit hyperactivity disorder; $\# p<0.01$ compared with adjustment disorder with mood disturbance.

tively associated with race ( $p \leq 0.02$ ) (higher in blacks) (Table $2)$. The triglyceride values were positively associated with Quetelet Index, alcohol (yes/no), the amount of alcohol, substance abuse, cigarettes (yes/no), and the number of cigarettes (Table 2).

On multivariate analysis, total cholesterol value was inversely associated with substance abuse and positively associated with race (higher in blacks) (Table 3 ). Substance abuse and race accounted for $5 \%$ of the variance of total cholesterol value $(p=$ $0.006)$. The triglyceride values were positively associated with cigarette use and Quetelet Index, were higher in whites, and were higher in boys (Table 3). A total of $10 \%$ of the variance of triglyceride values could be accounted for $(p=0.0004)$ (Table 3).

Associations of suicide attempts and suicide ideation with psychiatric diagnosis groups and with total plasma cholesterol levels. After covariance adjusting for age, race, sex, Quetelet Index, alcohol use, cigarette smoking, and substance abuse in the three major psychiatric diagnosis groups, children with adjustment disorders [who had the lowest adjusted mean total cholesterol value $(4.16 \mathrm{mmol} / \mathrm{L})]$ and children with affective disorders (least square mean total cholesterol value, $4.55 \mathrm{mmol} /$ L) were much more likely ( $p=0.003$ and $p=0.003$, respectively) to be suicidal or have suicide ideation than children with disruptive behavior whose least square mean cholesterol value was 4.47 $\mathrm{mmol} / \mathrm{L}$. Of children with disruptive behavior, $54 \%$ had attempted suicide or had suicide ideation compared with $81 \%$ of those with adjustment disorders and $79 \%$ of those with affective disorders (Fig. 5) $\left(\chi^{2}=13.4, p=0.001\right)$. Within the affective disorders group and in the group with adjustment disorders there were no differences $(p>0.1)$ in total cholesterol value, triglyceride value, age, Quetelet Index, alcohol use, cigarette smoking, or substance abuse between the children who were suicidal or had suicide ideation versus those who were not suicidal. How- ever, in the group of children with disruptive behavior, children who were suicidal or had suicide ideation were older $(p=0.01)$, more likely to abuse alcohol $(p=0.0006)$, smoked more $(p=$ $0.006)$, and were more likely to abuse drugs $(p=0.03)$.

We then examined whether suicide tendency differed among the nine major diagnostic subgroups within the three major psychiatric diagnoses (Table 1). To do this examination, we assessed whether differences existed in an arbitrarily developed suicide index among diagnostic subgroups (Fig. 6). Children with adjustment disorders and concomitant mood disturbance (depression), the group with the lowest covariance adjusted mean total cholesterol value $(4.03 \mathrm{mmol} / \mathrm{L})$, had much higher suicide indices than those in children with attention deficit hyperactivity disorder $(p=0.005)$ or oppositional defiant disorders $(p=$ 0.009 ), groups that respectively had higher least square mean total cholesterol values of 4.45 and $5.12 \mathrm{mmol} / \mathrm{L}(p=0.0003)$ (Fig. 6).

Lipids and axis II diagnoses. Axis II diagnoses included developmental disorders $(n=45)$, personality disorders $(n=67)$, and no diagnoses $(n=108)$. Plasma cholesterol and triglyceride values did not differ $(p>0.1)$ by axis II diagnosis.

Association of pharmacologic agents with total cholesterol and triglyceride values. Five major drug groups were used in these children: antidepressants $(n=26)$, antihypertensives $(n=4)$, mood stabilizers $(n=16)$, neuroleptics $(n=22)$, and stimulants $(n=16)$. Covariance-adjusted total cholesterol and triglyceride values did not differ among any of these five groups $(p>0.1)$.

\section{DISCUSSION}

Whereas bottom decile total cholesterol value was not overrepresented among children with psychiatric illness compared with healthy schoolchild controls, patients had a striking enrichment with hypertriglyceridemia. Triglyceride levels did not, however, differ by psychiatric diagnosis group. Obesity, a known determinant of triglycerides (28), may have contributed to the hypertriglyceridemia because Quetelet Indices were much higher $(p \leq$ 0.003 ) in patients than controls. Alcohol, known to contribute to elevated triglyceride levels (29), and cigarette smoking and substance abuse, all strong positive correlates of triglyceride levels in these patients, could also have contributed to their hypertriglyceridemia. Fowkes $e t$ al. (18) have reported a positive correlation of triglyceride values with hostile acts and domineering attitude. We have shown that high triglyceride values may be a determinant of symptoms of depression $(15,16)$. We have postulated $(15,16)$ that lowering triglyceride values with resultant reduced serum viscosity may lead to improved cerebral perfusion and oxygenation, with subsequent amelioration of symptoms of depression. Treatment of hypertriglyceridemia has also been reported to improve scores on dementia screening tests in elderly patients with cerebral vascular disease (30). This improvement may be the result of reductions in the elevated serum viscosity and secondary segmental cerebral hypoxia (30) associated with severe hypertriglyceridemias. Although group triglyceride values in the pediatric patients of the current study were sharply skewed upward to values above healthy schoolchildren controls, the patients did not have severe hypertriglyceridemia [ $>5.65 \mathrm{mmol} /$ L (15, 16)] (Fig. 7). Whether the neurobehavioral correlates of high serum triglyceride values $(15,16,18,30)$ overlap with or are distinct from those associated with low serum cholesterol concentration is presently unknown and will require prospective study for explanation.

In the current study, there were major associations of psychiatric diagnoses with plasma cholesterol value and with suicide index. Children with the lowest covariance adjusted mean cholesterol value (adjustment disorders with depression, $4.03 \mathrm{mmol} /$ L) were twice as likely to have attempted suicide or to have suicide ideation $(p<0.01)$ than those with disruptive disorder and attention deficit (total cholesterol value, $4.45 \mathrm{mmol} / \mathrm{L}$ ) and disruptive disorder (oppositional defiant) (total cholesterol value, 

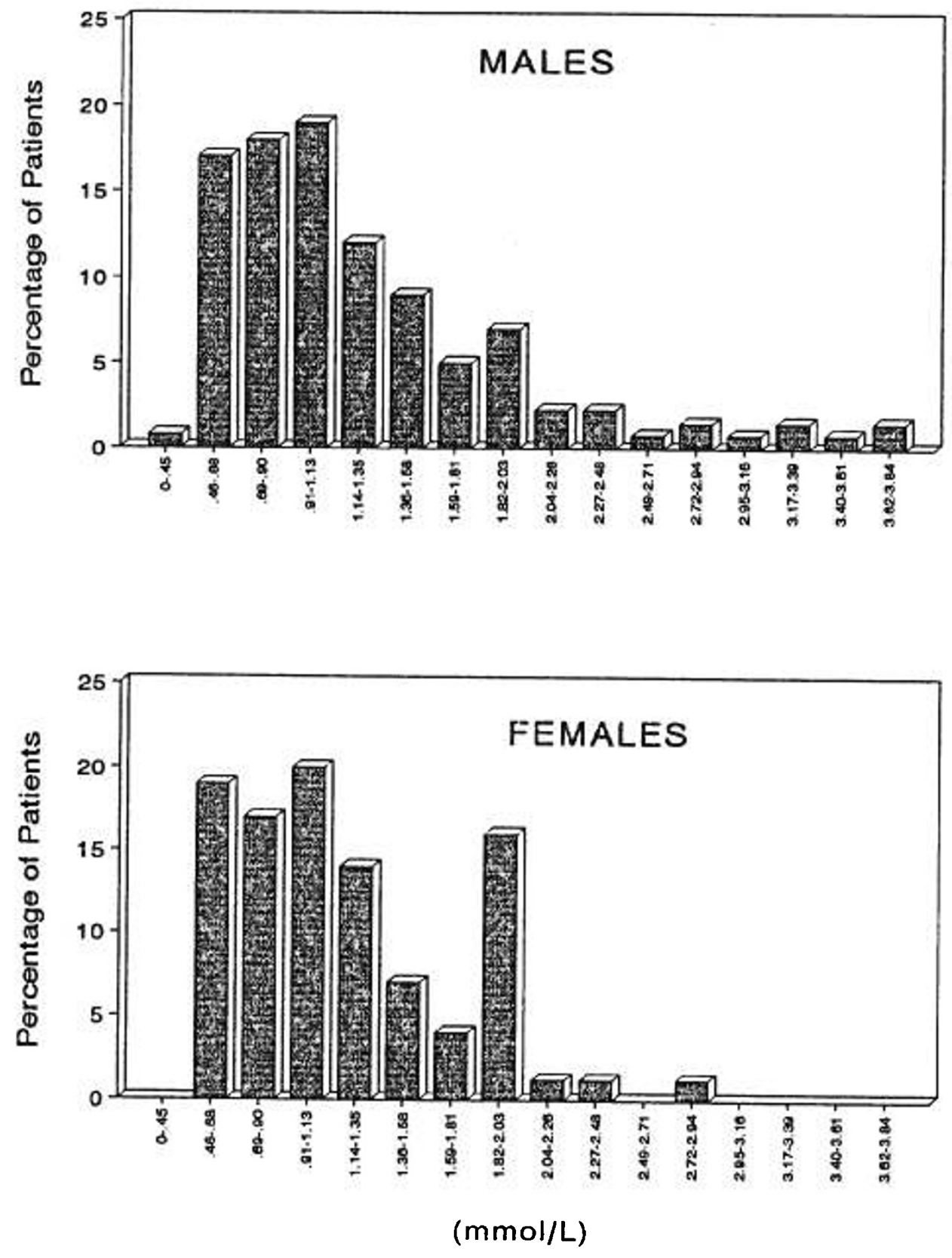

Fig. 7. Frequency distribution in male and female pediatric psychiatric inpatients for plasma triglyceride levels $(\mathrm{mmol} / \mathrm{L})$.

$5.12 \mathrm{mmol} / \mathrm{L})$. The association of low plasma cholesterol level with affective disorders has also been seen by us $(9,10)$ in 203 adult patients hospitalized with affective disorders who were five times more likely than population controls to have cholesterol values less than $4.14 \mathrm{mmol} / \mathrm{L}$. Morgan et al. (11) have reported higher depressive symptom scores in men 70 years of age or older whose plasma cholesterol value was less than $4.14 \mathrm{mmol} /$ L. They also reported significant correlations between depressive symptom scores (including weight and appetite loss) and plasma cholesterol value but also observed that "... the association between cholesterol concentration and depressive symptoms was independent of reported or measured change in weight, which suggests that low cholesterol is not entirely the consequence of depression related malnutrition." Subnutrition with attendant hypocholesterolemia may be a component of depression-mediated morbidity, or, as noted by Morgan et al. (11), "... it is possible that both depression and low cholesterol are indicators of poor health status." Because the children of our current study were relatively obese as measured by Quetelet Index, subnutrition or malnutrition were unlikely. Another factor beyond nutrition that possibly contributed to low cholesterol levels, however, was substance abuse, which, in univariate and multivariate analyses, was an independent, inverse explanatory variable for plasma cholesterol level. However, by itself, correlation falls well short of establishing causal relationship, and it remains to be determined whether substance abuse might lower plasma cholesterol level through hepatotoxic or other "pharmacologic" avenues. Substance abuse and alcohol intoxication are also strong positive determinants of suicidal behavior in adolescents (24). It is possible that both substance abuse and low plasma cholesterol level in children are both attributable to a third, unmeasured factor, or, however unlikely, that substance abuse is a result of a low plasma cholesterol level. This latter possibility might be examined by prospective follow-up with a focus on psychopathologic disorder, alcohol use, and substance abuse in schoolchildren in epidemiologic studies whose initial plasma total cholesterol value was less than $3.3 \mathrm{mmol} / \mathrm{L}(<128 \mathrm{mg} / \mathrm{dL})(28,29)$.

Low plasma cholesterol level has not been a uniform finding in patients with affective disorders (31-33). Lang and Haits (31) reported that serum cholesterol levels were higher in patients 
with major depression than in controls. Oxenkrug et al. (32) reported that age and sex influenced serum cholesterol values in depressed patients but that the diagnosis of major depression did not. Feder (33) has reported that among patients with major depression, histories of anxiety disorders were associated with increased serum cholesterol levels.

The mechanisms by which low plasma cholesterol levels might contribute to affective disorders, psychopathologic disorders, and suicide tendencies (4) remain unknown and highly speculative. It is possible that low plasma cholesterol levels might contribute to low levels of brain serotonin, which is postulated to inhibit harmful behavioral impulses (34). However, we have previously reported $(35,36)$ that subjects heterozygous for familial hypobetalipoproteinemia who have lifelong, extraordinarily low plasma cholesterol values (usually lower than $2.59 \mathrm{mmol} / \mathrm{L}$ ), live an average 12 y longer than their birth cohort and are not characterized by suicide and violent death. This relatively common autosomal dominant disorder, occurring in approximately 1 of 500 unselected kindreds (36), can be taken as an experiment of nature, suggesting that heritable low plasma total cholesterol level is associated with reduced all-cause mortality. Twelve-vear follow-up studies of 350.977 men who had been screened for Multiple Risk Factor Intervention Trial revealed a J-shaped relationship between serum cholesterol and all-cause mortality. with the inflection point of the $\mathrm{J}$ at $3.62 \mathrm{mmol} / \mathrm{L}(140 \mathrm{mg} / \mathrm{dL})$. below which crude death rates rose with decreasing serum cholesterol values and above which crude death rates rose with increasing serum cholesterol values (2). However, as reported by Neaton e't al. (2), "based on a proportional hazards regression model ... a serum cholesterol level of $3.15 \mathrm{mmol} / \mathrm{L}(122 \mathrm{mg} /$ dL) was associated with a minimum risk of death."

Whether hypocholesterolemia in some pediatric psychiatric diagnosis groups may be causally associated with the likelihood of psychiatric hospitalization, suicide tendency, or both remains to be determined by prospective study, as does the broad association of hypertriglyceridemia with pediatric psychiatric hospitalization. Like Neaton $e t$ al. (2) did in adults, we postulate that hypocholesterolemia in children hospitalized because of psychiatric disease may be a consequence of affective disorder-mediated behavior change.

\section{REFERENCES}

1. Lindberg G, Rastam L, Gullberg B, Eklund GA 1992 Low serum cholesterol concentration and short term mortality from injuries in men and women. BMJ 305:277-279

2. Neaton JD, Blackburn : I, Jacobs D. Kuller L, Lee DJ, Sherwin R, Chir B, Shih H, Stamler J, Wentworth D 1992 Serum cholesterol level and mortality findings for men screened in the multiple risk factor intervention trial. Arch Intern Med 152:1490-1500

3. Jacobs D, Blackburn H. Higgins M, Reed D, Iso H, McMillian G, Neaton J, Nelson J, Potter J, Rifkind B, Rosseau J, Shekelle R. Yusuf S 1992 Report of the conference on low blood cholesterol: mortality associations. Circulation 86:1046-1060

4. Muldoon MF, Rossouw JE, Manuck SB, Glueck CJ, Kaplan JR, Kaufmann PG 1993 Low or lowered cholesterol and risk of death from suicide and trauma. Metabolism 42(suppl 1):45-56

5. Muldoon MF, Manuck SB, Matthews KA 1990 Effects of cholesterol lowering on mortality: a quantitative review of primary prevention trials. BMJ 301:309-314

6. Muldoon MF, Manuck SB, Matthews KA 1991 Does cholesterol lowering increase non-illness-related mortality? Arch Intern Med 151:1453-1454
7. Wysowski DK Gross TP 1990 Deaths due to accidents and violence into recent trials of cholesterol-lowering drugs. Arch Intern Med 150:2169-2172

8. Sletten IW. Nilsen JA. Young RC. Anderson JT 1964 Blood lipids and behavior in mental-hospital patients. Psychosom Med 26:261-266

9. Glueck CJ. Sanghvi VR. Laemmle P. Unger L, McCray C. Tracy T, Speirs J, Lang J, Tieger M. Kunkel R 1990 Lack of concordance in classification of coronary heart disease risk: high-risk HDL cholesterol $<35 \mathrm{mg} / \mathrm{dl}$ in subjects with desirable total serum cholesterol $<200 \mathrm{mg} / \mathrm{dl}$. J Lab Clin Med 116:377385

10. Glueck CJ, Tieger M, Kunkel R. Tracy T, Speirs J 1993 Hypocholesterolemia affective disorders, and suicide? Clin Res 41:123A(abstr)

11. Morgan RE, Palinkas LA, Barrett-Connor EL, Wingard DL 1993 Plasma cholesterol and depressive symptoms in older men. Lancet 341:75-79

12. Virkkunen M, Penttinen H 1984 Serum cholesterol in aggressive conduct disorder: a preliminary study. Biol Psychiatry 19:435-439

13. Virkkunen M 1979 Serum cholesterol in antisocial personality. Neuropsychobiology 5:27-30

14. Virkkunen M 1983 Serum cholesterol levels in homicidal offenders. Neuropsychobiology 10:65-69

15. Tieger M. Kunkel R. Tracy T. Speirs J, Streicher P. Illig E, Vandegrift J, Glueck CJ 1991 Amelioration of depression by treatment of severe hypertriglyceridemia. Clin Res 39:464A(abstr)

16. Glueck CJ, Tieger M, Kunkel R. Tracy T, Speirs J, Streicher P. Illig E 1993 Improvement in symptoms of depression and in an index of life stressors accompany treatment of severe hypertriglyceridemia. Biol Psychiatry $34: 240-252$

17. Beck AT, Beck RW 1972 Screening depressed patients in family practice: rapid technique. Postgrad Med 52:31-85

18. Fowkes FGR, Leng GC, Donnan PT, Deary IJ, Riemersma RA, Housley E 1992 Serum cholesterol, triglycerides, and aggression in the general population. Lancet 340:995-998

19. Holinger PC 1987 Violent Deaths in the United States. The Guilford Press, New York

20. Klebba AJ 1979 Comparison of trends for suicide and homicide in the United States, 1900-1976. In: Hays JR. Roberts TK. Solway KS (eds) Violence and the Violent Individual. SP Medical \& Scientific Books, New York, pp 127 148

21. Porterfield AL 1960 Traflic fatalities, suicide, and homocide. Am Socio Rev 25:897-901

22. Blumenthal SJ 1990 Youth suicide: risk factors, assessment, and treatment of adolescent and young adult suicidal patients. Psychiatr Clin North Am 13:511-556

23. Low BP, Andrews SF 1990 Adolescent suicide. Med Clin North Am 74:12511264

24. Crumley FE 1990 Substance abuse and adolescent suicidal behavior. JAMA 263:3051-3056

25. Garrison CZ 1989 The study of suicidal behavior in the schools. Suicide Life Threat Bchav 19:120-130

26. Snedecor GW, Cochran WG 1980 Statistical Methods, 7th ed. lowa State University Press, Ames, IA

27. SAS Procedures Guide 1988 SAS Institute, Cary, NC

28. Laskarzewski PM, Morrison JA, Mellies MJ, Kelly K. Gartside PS, Khoury P. Glueck CJ 1980 Relationships of measurements of body mass to plasma lipoproteins in schoolchildren and adults. Am J Epidemiol 111:395-407

29. Glueck CJ, Heiss G, Morrison JA, Khoury P. Moore M 1981 Alcohol intake. cigarette smoking, and plasma lipids and lipoproteins in 12 year old to 19 year old children. The Collaborative Lipid Research Clinics Prevalence Study. Circulation 64:48-56

30. Rogers RL. Meyer JS, McClintic K, Mortel KF 1989 Reducing hypertriglyceridemia in elderly patients with cerebrovascular disease stabilizes or improves cognition and cerebral perfusion. Angiology 40:260-269

31. Lang S, Haits G 1968 Blutserum cholestinwerte bei depression. Das Deutsche Gesundheitswesen 23:82-84

32. Oxenkrug GF, Branconnier RJ, Harto-Truax N. Cole JO 1983 Is serum cholesterol a biological marker for major depressive disorder? Am J Psychiatry 140:920-921

33. Feder R 1993 High cholesterol levels in patients with panic disorder [letter] Am J Psychiatry 150:527

34. Engelberg if 1992 Low serum cholesterol and suicide. Lancet 339:727-729

35. Glueck CJ, Gartside P, Fallat RW, Sielski J, Steiner PM 1976 Longevity syndromes: familial hypobeta and familial hypoalphalipoproteinemia. J Lab Clin Med 88:941-956

36. Glueck CJ, Gartside PS, Mellies MJ, Steiner PM 1977 Familial hypobetalipoproteinemia: studies in 13 kindreds. Trans Assoc Am Physicians 90:184203 